\title{
The synthesis of new thiosubstituted butadienes, butenynes and butatrienes
}

\author{
Cemil İbiş* and Neslihan Beyazit \\ Istanbul University, Faculty of Engineering, Department of Chemistry, Istanbul, 34320, Turkey \\ E-mail: ibiscml@,istanbul.edu.tr
}

\begin{abstract}
By treatment of the pentachlorobutadiene $\mathbf{1}$ and of the tetrachlorobromobutadiene $\mathbf{9}$ with thiolates in ethanol, a very fast and extensive replacement of chlorine was observed even at room temperature. The reaction of $\mathbf{1}$ with three molar equivalents of thiolates lead to butadienes with two, three, four and five organylthio groups. Tris(thio)-substituted butadienes 3a-c tetrakis (thio)-substituted butadiene $4 \mathbf{a}$ were treated with potassium tert-butoxide to form tris(thio)substituted butatrienyl halide compounds 12a-c and tetrakis(thio)-substituted butatriene 14a, respectively. The butatrienyl halides $\mathbf{1 2 a - c}$ obtained partly isomerizes to give butenynes 13a-c at room temperature and without catalyst.
\end{abstract}

Keywords: Thiosubstituted butadienes, butenynes, butatrienes, thiols, $\mathrm{HCl}$ elimination

\section{Introduction}

Polyhalogenobutadienes have been used as model substrates for studying of the process of vinylic substitution $\left(\mathrm{S}_{\mathrm{N}} \mathrm{Vin}\right)$. There are various possibilities of the use of these compounds for the sythesis of numerous polyfunctional products. The reactions of $2 \mathrm{H}$ - and $1 \mathrm{H}$ pentachlorobutadienes, 1,3-di- $H$ - and 2,3-di- $H$-hexachlorobutenes with some thiols are well documented and mono- and polythiosubstituted compunds, mostly butadiene derivatives, have been obtained by this way. ${ }^{1-7}$

It is known that some thiosubstituted butadienes exhibit high biological activity. Industrial research has long focused on biological applications of thiosubstituted compounds as insectisides, herbicides, fungicides, and bactericides. ${ }^{8}$ 5-lipoxygenase inhibition effect of 1-thiosubstituted butadienes also reported. ${ }^{9}$ Butenynes and butatrienes are very valuable compounds for their use in polymer chemistry. Unlike alkanes and most alkenes, cumulenes tends to be rigid, which makes them appealing for molecular nanotechnology. ${ }^{10-12}$ Thiosubstituted compounds are used as electronic conductors, ferromagnets, electron-accepting supramolecules, optical materials in material chemistry and as stabilizers in polimer chemistry. ${ }^{13}$ 
Schmidt et al. have synthesised thiosubstituted butatrienes and 1,3-butadienes from the reaction of a tetrakis(pyridinium)-substituted butadiene with some thiolates. ${ }^{14}$ In an earlier study, Block et al. have used perchalcogenohydrocarbons as starting material to obtain perthio-1,3butadienes, -1-buten-3-ynes and -1,2,3-butatrienes. ${ }^{15}$ In this work, we report another efficient method for the synthesis of novel thiosubstituted butadiene, butenyne and butatriene compounds.

\section{Results and Discussion}

The reactions of $2 \mathrm{H}$-pentachlorobutadiene with three molar equivalents of thiols in the presence of $\mathrm{NaOH}$ in ethanol at room temperature were carried out to give bis-, tris-, tetrakis- and pentakis(thio)-substituted butadienes, 2d, 3a-c, 4a, 5a and tetrakis(thio)-substituted butenyne 6d. New mono(thio)-substituted butenyne compunds $\mathbf{7 a}, \mathbf{7 c}, \mathbf{7 e - i}$ were obtained from the reactions of $2 \mathrm{H}$-pentachlorobutadiene and $\mathbf{1 0 e - i}$, from the reactions of $2 H$-1,1,3,4-tetrachloro-4bromobutadiene with one molar equivalent of thiols at the same reaction conditions. Monothiosubstituted polyhalogenobutadienes 8a, 8c, 8e-i and $11 \mathbf{h}$ were synthesized from the bromination of mono(thio)-substituted butenyne compounds in an apolar solvent (Scheme 1).

Compounds 3a, 4a and 5a were obtained from the reaction of $2 \mathrm{H}$-pentachlorobutadiene with three molar equivalents of thiol a. In the possible reaction mechanism of 5a, it is thought that perchlorobutenyne formed by $\mathrm{HCl}$ elimination from $2 \mathrm{H}$-pentachlorobutadiene firstly and then tetrakis(thio)-substituted butenyne and tetrakis(thio)-substituted butatriene intermediates were occurred via addition of four molar of thiol a to perchlorobutenyne. These intermediates both were stabilized by mesomery. In the last step of the mechanism, 5a was obtained by addition of one molar of thiol a to tetrakis(thio)-substituted butenyne or tetrakis(thio)-substituted butatriene.

${ }^{1} \mathrm{H}-\mathrm{NMR}$ spectra of $\mathbf{2 d}$, 3a-c, $4 \mathbf{a}$ and 5a exhibited the presence of vinyl proton as a singlet at approximately $6.50 \mathrm{ppm}$. The mass spectrum of $\mathbf{3 a}$ confirmed the estimated structure; two main peaks were observed at $\mathrm{m} / z 683.62$ and 648.77 corresponding to [M] ${ }^{+}$and $[\mathrm{M}-\mathrm{Cl}]^{+}$, respectively. The IR spectra of butenyne derivatives $6 \mathbf{d}, 7 \mathbf{a}, 7 \mathbf{c}, 7 \mathbf{e}-\mathbf{i}$ and 10e-i showed the characteristic strong band at 2147 and $2152 \mathrm{~cm}^{-1}$ for $\mathrm{C} \equiv \mathrm{C}$ group. In the ${ }^{13} \mathrm{C}-\mathrm{NMR}$ spectra of these compounds, two alkyne carbons provide chemical shift values around 85.11 and $92.07 \mathrm{ppm}$. In the ESI-MS spectrum of 10h showed a molecular ion peak at $\mathrm{m} / \mathrm{z} 455.93$ and the fragmentation of molecular ion peak at $m / z 375$ corresponding to the loss of a bromine atom. New thiosubstituted polyhalobutadiene compounds $8 \mathbf{8}, \mathbf{8 c}, \mathbf{8 e - i}$ and $11 \mathbf{e}-\mathbf{i}$ were formed by the electrophilic addition of $\mathrm{Br}_{2}$ to butenynes. It is evidence for succesful bromination reaction that the IR spectra of these compounds showed no absorbtion band around 2147 or $2152 \mathrm{~cm}^{-1}$. 

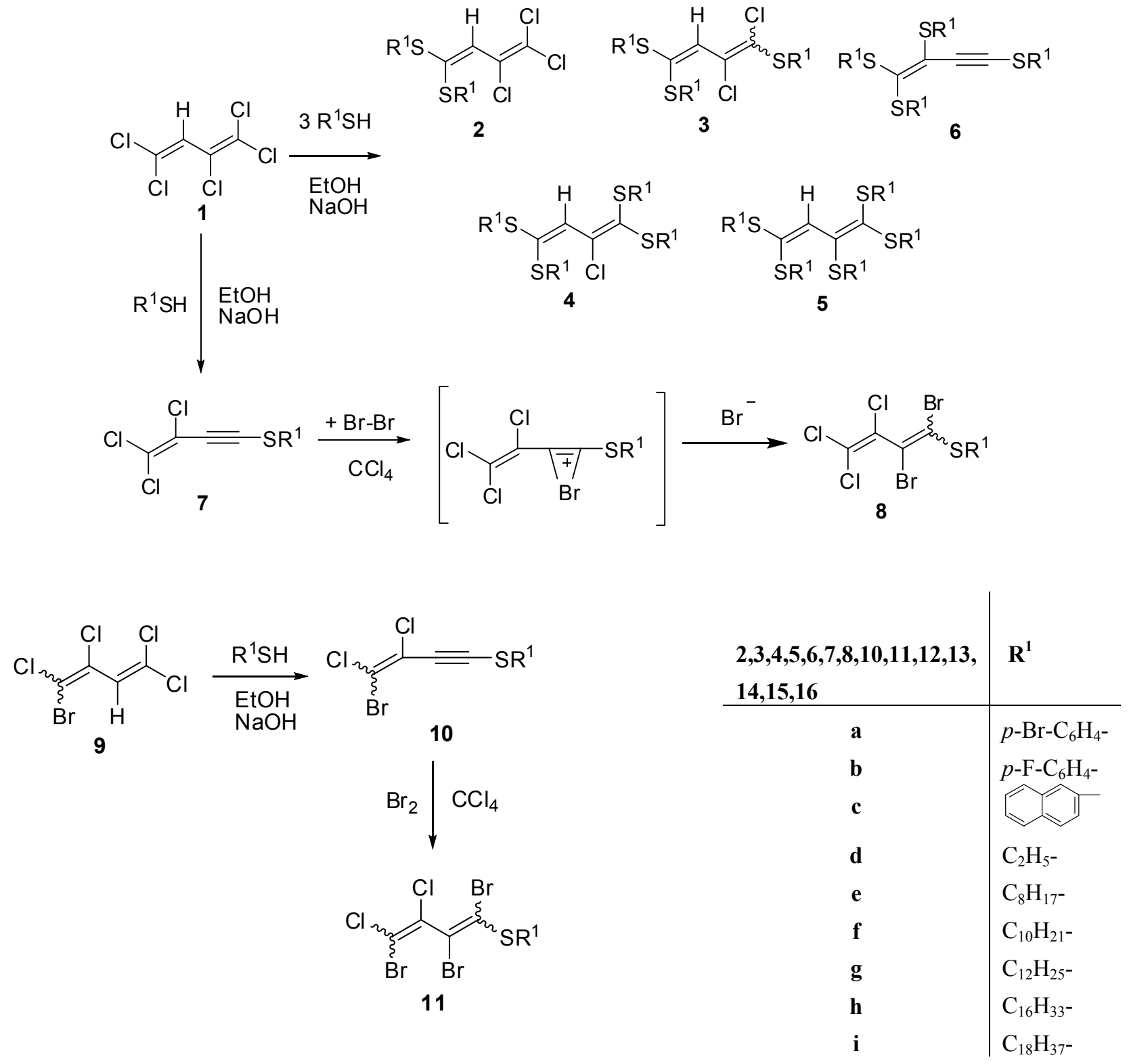

Scheme 1. General synthesis methods of butadienes and butenynes.

Tris(thio)-substituted butatriene compounds 12a-c were formed by $\mathrm{HCl}$ elimination in the presence of potassium tert-butoxide from tris(thio)-substituted butadienes 3a-c. These butatriene compounds 12a-c partly isomerize to the tris(thio)-substituted butenynes 13a-c even at room temperature, in a solvent medium. Tetrakis(thio)-substituted butatriene 14a, obtained from 4a by $\mathrm{HCl}$ elimination, is more stable than 12a-c. This stability was proved by IR spectrum of 14a that there was no typical absorption band at $2142-2157 \mathrm{~cm}^{-1}$ region corresponding to $\mathrm{C} \equiv \mathrm{C}$ group. Addition of $\mathrm{I}_{2}$ to butatriene compounds 12a and 14a were performed in apolar solvent at room temperature to give 15a and 16a. Electrophilic addition reaction mechanism proceeds via an iodonium cation (Scheme 2). 


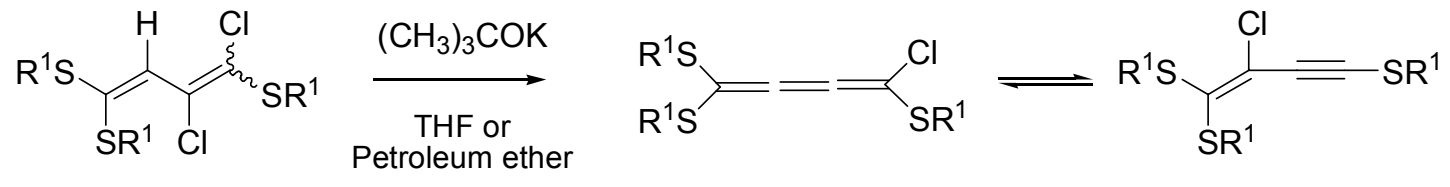

3

4

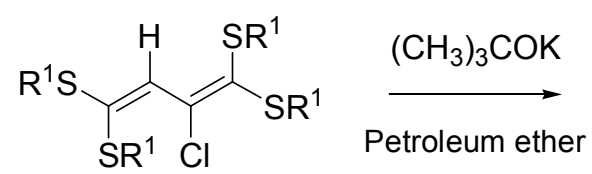

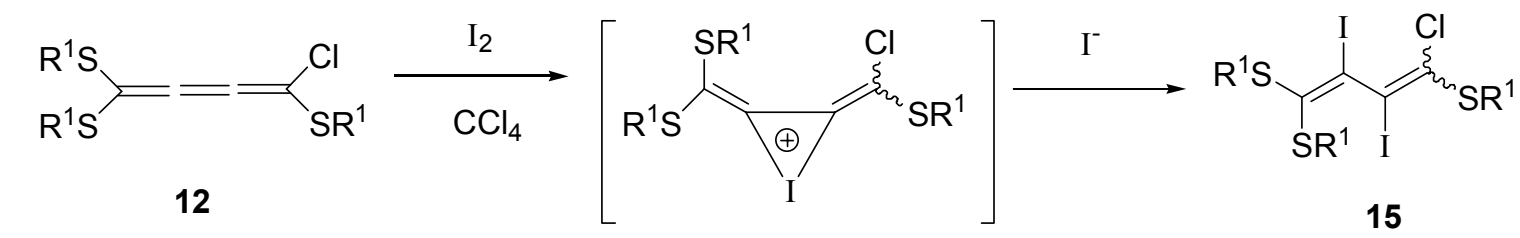

12

13

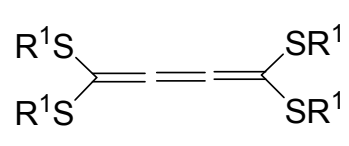

14

Scheme 2. The synthesis and iodination of butatriene compounds.

The solvolysis of butatrienyl halides gives the ambident vinyl cation which has positive charge on a disubstituted carbon in both mesomeric structures ( $x$ and $y$ ). The possible isomerization mechanism of 1-chloro-1,4,4-tris(4-methylphenylthio)butatriene have been explained in the previous study by our group (Scheme 3$).^{5}$

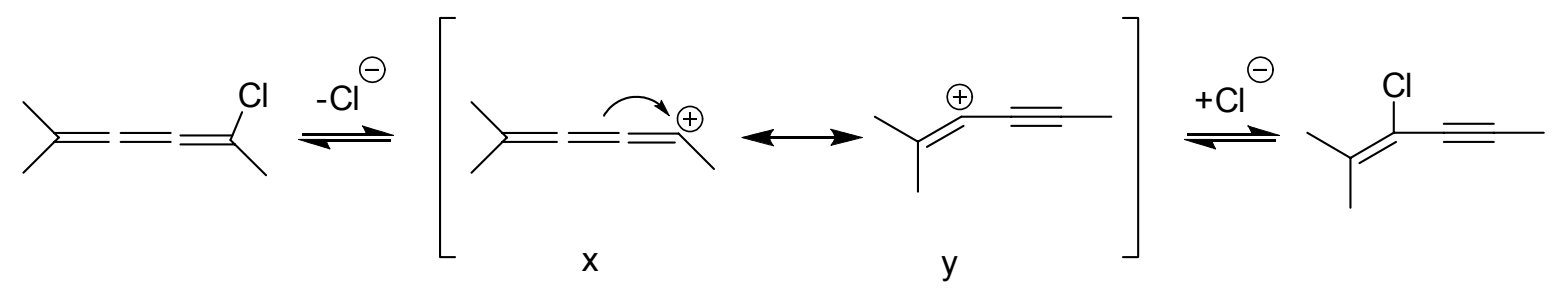

Scheme 3. The possible isomerization mechanism of butatrienyl halides to butenynes.

12a and 12c obtained under the action of potassium tert-butoxide, are stable solid compounds. The peaks at 2037 and $2043 \mathrm{~cm}^{-1}$ in the IR spectra of 12a and 12c was assigned to $C=C=C=C$ stretching vibration, respectively. 12b, yellow oily compound, easily isomerizes to 1-buten-3ynes $\mathbf{1 3 b}$ at room temperature without catalyst. IR spectrum of $\mathbf{1 2 b}$ showed $\mathrm{C} \equiv \mathrm{C}$ stretching band at $2143 \mathrm{~cm}^{-1}$ next to butatriene band at $2043 \mathrm{~cm}^{-1}$. However, in the solvent medium, stable butatrienyl halides 12a and 12c are solvolyzed to give the ambident allenyl cation which isomerizes to butenyne compounds $\mathbf{1 3 a}$ and $\mathbf{1 3} \mathbf{c}$.

12a-c cannot be isolated from the isomeric mixture by the chromatographic techniques. IR, Mass spectra and elemental analyses results confirmed that these compounds are the isomeric mixture 
of tris(thio)-substituted butatriene and tris(thio)-substituted butenyne. IR spectrum of the isomeric mixture of 12a and 13a showed characteristic absorption bands at 2037 and $2142 \mathrm{~cm}^{-1}$ due to $\mathrm{C}=\mathrm{C}=\mathrm{C}=\mathrm{C}$ and $\mathrm{C} \equiv \mathrm{C}$ groups, respectively. The ESI-MS in the positive ion mode of the mixture of compounds 12a and 13a, displays an ion of $\mathrm{m} / \mathrm{z} 648.84$ assigned to the protonated molecular ion peak $[\mathrm{M}+\mathrm{H}]^{+}$. The major ion of $\mathrm{m} / \mathrm{z} 459.90$ in the ESI-MS/MS of 12a and 13a indicates the loss of $-\mathrm{SC}_{6} \mathrm{H}_{4} \mathrm{Br}$ group. The presence of isomer mixture was also confirmed by the ${ }^{13} \mathrm{C}-\mathrm{NMR}$ spectrum, in which more signals than expected chemical shifts were observed and two internal alkynes appeared at 86.24 and $93.19 \mathrm{ppm}$.

Thiosubstituted butadiene and butenyne compounds showed maximum absorption of the range 244-266 $\mathrm{nm}$ in $\mathrm{CHCl}_{3}$, the maximum absorption wavelenghts of butatriene compounds were observed between $367-392 \mathrm{~nm}$.

\section{Experimental Section}

General. Melting points were measured on Buchi B-540 capillary apparatus and are uncorrected. IR-spectras were recorded on Shimadzu FTIR-8101. NMR spectra were recorded on Varian Unity Inova $500 \mathrm{MHz}$. Mass spectra were obtained on a Thermo Finnigan LCQ Advantage MAX LC/MS/MS Spectrometer using ion-trap mass analyzer for both APCI or ESI source. UV spectra were recorded on UV-VIS Spectrophotometer TU-1901. Microanalyses were obtained by using a Carlo-Erba 1110 element analyser. Thin-layer chromatography (TLC) : E. Merck silica gel $60 \mathrm{~F}_{254}$ foils. Column chromatography: Silica gel 60 ( particle size 0.063-0.20 mm, E. Merck ).

\section{General procedure 1}

$2 \mathrm{H}$-pentachlorobutadiene $1(1 \mathrm{~g}, 4.4 \mathrm{mmol})$ and thiols $(13.2 \mathrm{mmol})$ were stirred in a mixture of EtOH $(30 \mathrm{~mL})$ and aqueous solution of $\mathrm{NaOH}(1.2 \mathrm{~g}$ and $8 \mathrm{ml}$ water) for $30 \mathrm{~min}$. at room temperature. Ether was added to the reaction mixture and the organic layer was separated, washed with water $(4 \times 30 \mathrm{~mL})$, and dried with $\mathrm{MgSO}_{4}$. The solvent was evaporated and the residue was purified by column chromatography over silica gel (petroleum ether/chloroform or petroleum ether).

1,2-Dichloro-1,4,4-tris(4-bromophenylthio)-1,3-butadiene (3a). Yield 40\%; White solid, mp: 80-82 ${ }^{\circ} \mathrm{C} . \mathrm{R}_{f}$ (Petroleum ether): 0.35; IR $\left(\mathrm{KBr}, \mathrm{cm}^{-1}\right): 1580(\mathrm{C}=\mathrm{C}), 3100\left(=\mathrm{C}-\mathrm{H}_{\text {arom }}\right)$; UV$\operatorname{vis}\left(\mathrm{CHCl}_{3}\right): \lambda_{\max }(\log \varepsilon)=244 \mathrm{~nm}(5.1), 266(5.0), 330(4.8) ;{ }^{1} \mathrm{H} \mathrm{NMR}\left(499.83 \mathrm{MHz}, \mathrm{CDCl}_{3}\right): \delta$ 6.49 (s, $1 \mathrm{H}$, vinyl-H), 7.02-7.42 (m, $\left.12 \mathrm{H}, \mathrm{H}_{\text {arom }}\right) ;{ }^{13} \mathrm{C} \mathrm{NMR}\left(125.68 \mathrm{MHz}, \mathrm{CDCl}_{3}\right): \delta 130.91$, $131.01,131.38,131.45,132.81,133.05,133.34,133.40\left(\mathrm{CH}_{\text {arom }}\right), 121.48,122.12,122.26,126.32$, $126.69,130.32,130.52,130.91,139.69$ ( $\mathrm{C}_{\text {arom }}$ and $\left.\mathrm{C}_{\text {butad }}\right)$; $\mathrm{MS}(+\mathrm{ESI}): \mathrm{m} / z 683.62[\mathrm{M}]^{+}, 648.77$ [M-Cl] $]^{+} \mathrm{C}_{22} \mathrm{H}_{13} \mathrm{~S}_{3} \mathrm{Cl}_{2} \mathrm{Br}_{3}$ (M, 684.15). Calcd. C, 38.62; H, 1.91; S, 14.06. Found C, 38.61; H, $1.85 ; \mathrm{S}, 14.66$. 
2-Chloro-1,1,4,4-tetrakis(4-bromophenylthio)-1,3-butadiene (4a). Yield 12\%; Yellow solid, mp: $135-137^{\circ} \mathrm{C} . \mathrm{R}_{f}\left[\right.$ Petroleum ether/ $\left.\mathrm{CCl}_{4}(1: 1)\right]: 0.42 ; \mathrm{IR}\left(\mathrm{KBr}, \mathrm{cm}^{-1}\right): 1502,1563(\mathrm{C}=\mathrm{C}), 3079$ $\left(=\mathrm{C}-\mathrm{H}_{\text {arom }}\right) ;{ }^{1} \mathrm{H}$ NMR $\left(499.83 \mathrm{MHz}, \mathrm{CDCl}_{3}\right): \delta 6.76\left(\mathrm{~s}, 1 \mathrm{H}\right.$, vinyl-H), 6.78-7.34 (m, $\left.16 \mathrm{H}, \mathrm{H}_{\text {arom }}\right)$; ${ }^{13} \mathrm{C}$ NMR $\left(125.68 \mathrm{MHz}, \mathrm{CDCl}_{3}\right): \delta 121.59,122.61,123.16,123.20,129.85,130.98,131.13$, $131.79,131.82,132.03,132.16,132.21,132.25,132.52,133.04,133.96,134.49,135.41$ ve $139.61\left(\mathrm{CH}_{\text {arom }}, \mathrm{C}_{\text {arom }}\right.$ and $\left.\mathrm{C}_{\text {butad }}\right) ; \mathrm{C}_{28} \mathrm{H}_{17} \mathrm{~S}_{4} \mathrm{ClBr}_{4}(\mathrm{M}, 836.76)$. Calcd. C, 40.19; H, 2.05; S, 15.33. Found C, 40.87; H, 1.95; S, 15.59 .

1,1,2,4,4-Pentakis(4-bromophenylthio)-1,3-butadiene (5a). Yield 6\%; Yellow solid, mp:157$159^{\circ} \mathrm{C} . \mathrm{R}_{f}$ [Petroleum ether $\left./ \mathrm{CHCl}_{3}(1: 1)\right]: 0.13$, IR $\left(\mathrm{KBr}, \mathrm{cm}^{-1}\right): 1561(\mathrm{C}=\mathrm{C}), 3074\left(=\mathrm{C}-\mathrm{H}_{\text {arom }}\right) ;{ }^{1} \mathrm{H}$ NMR $\left(499.83 \mathrm{MHz}, \mathrm{CDCl}_{3}\right): \delta 6.34\left(\mathrm{~s}, 1 \mathrm{H}\right.$, vinyl-H), 6.81-7.44 (m, $\left.20 \mathrm{H}, \mathrm{H}_{\text {arom }}\right) ;{ }^{13} \mathrm{C}$ NMR (125.68 MHz, $\left.\mathrm{CDCl}_{3}\right): \delta 121.92,122.06,122.64,122.77,123.07,130.15,131.28,131.50,131.90$, 132.10, 132.27, 132.38, 132.51, 132.67, 133.20, 133.29, 134.06, 134.38, 135.17, 137.83, 141.31 $\left(\mathrm{CH}_{\text {arom, }} \mathrm{C}_{\text {arom }}, \mathrm{C}_{\text {butad }}\right) ; \mathrm{C}_{34} \mathrm{H}_{21} \mathrm{~S}_{5} \mathrm{Br}_{5}(\mathrm{M}, 989.39)$. Calcd. C, 41.28; H, 2.14; $\mathrm{S}, 16.20$. Found $\mathrm{C}$, 40.93; H, 2.05; S, 16.82.

1,2-Dichloro-1,4,4-tris(4-fluorophenylthio)-1,3-butadiene (3b). Yield 42\%; Oil, $\mathrm{R}_{f}$ [Petroleum ether/CHCl $\left.{ }_{3}(1: 1)\right]: 0.40$; IR (film, $\left.\mathrm{cm}^{-1}\right): 1589(\mathrm{C}=\mathrm{C}), 3068$ (=C-Harom); UV-vis $\left(\mathrm{CHCl}_{3}\right): \lambda_{\max }$ $(\log \varepsilon)=257 \mathrm{~nm}(4.6), 326(4.5) ;{ }^{1} \mathrm{H}$ NMR $\left(499.83 \mathrm{MHz}, \mathrm{CDCl}_{3}\right): \delta 6.48$ (s, $1 \mathrm{H}$, vinyl-H), 7.02$7.48\left(\mathrm{~m}, 12 \mathrm{H}, \mathrm{H}_{\text {arom }}\right) ;{ }^{13} \mathrm{C}$ NMR $\left(125.68 \mathrm{MHz}, \mathrm{CDCl}_{3}\right): \delta 116.15,116.32,116.58,116.65,116.76$, 116.83, 125.69, 127.03, 127.64, 128.36, 133.94, 135.19, 135.25, 135.71, 135.77, 135.98, 136.05, 142.56, 162.37, 164.13, $164.37\left(\mathrm{CH}_{\text {arom }}, \mathrm{C}_{\text {arom }}\right.$ and $\left.\mathrm{C}_{\text {butad }}\right)$; $\mathrm{MS}$ (+ESI): $\mathrm{m} / z 500.97[\mathrm{M}]^{+}, 464.96$

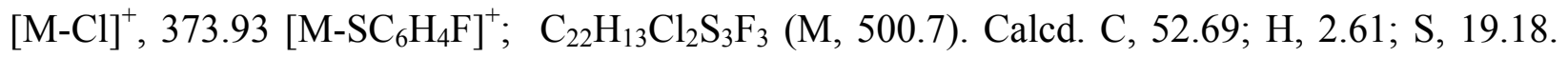
Found C, 53.19; H, 2.99; S, 18.14 .

1,2-Dichloro-1,4,4-tris(2-naphtylylthio)-1,3-butadiene (3c). Yield 34\%; White solid, mp: 143$145^{\circ} \mathrm{C} . \mathrm{R}_{f}\left[\right.$ Petroleum ether $\left./ \mathrm{CCl}_{4}(1: 1)\right]: 0.30$; IR $\left(\mathrm{KBr}, \mathrm{cm}^{-1}\right): 1580(\mathrm{C}=\mathrm{C}), 3050\left(=\mathrm{C}-\mathrm{H}_{\text {arom }}\right)$; UV$\operatorname{vis}\left(\mathrm{CHCl}_{3}\right): \lambda_{\max }(\log \varepsilon)=259 \mathrm{~nm}(4.9) ;{ }^{1} \mathrm{H} \operatorname{NMR}\left(499.83 \mathrm{MHz}, \mathrm{CDCl}_{3}\right): \delta 6.55(\mathrm{~s}, 1 \mathrm{H}$, vinyl-H), 7.25-7.84 (m, 21H, $\left.\mathrm{H}_{\text {arom }}\right) ;{ }^{13} \mathrm{C}$ NMR $\left(125.68 \mathrm{MHz}, \mathrm{CDCl}_{3}\right): \delta 126.71,126.79,126.86,126.96$, $127.09,127.17,127.80,127.95,127.99,128.36,128.61,129.02,129.11,129.23,129.66,129.75$, 129.88, 129.93, 130.11, 132.08, 132.29, 132.48, 132.99, 133.13, 133.19, 133.63, 133.74, 133.77, $142.11\left(\mathrm{CH}_{\text {arom }}, \mathrm{C}_{\text {arom }}\right.$ and $\left.\mathrm{C}_{\text {butad }}\right) ; \mathrm{MS}(+\mathrm{ESI}): \mathrm{m} / z 597.80[\mathrm{M}]^{+}, 560.97[\mathrm{M}-\mathrm{Cl}]^{+} ; \mathrm{C}_{34} \mathrm{H}_{22} \mathrm{Cl}_{2} \mathrm{~S}_{3}(\mathrm{M}$, 597.64). Calcd. C, 68.33; H, 3.71; S, 16.10. Found C, 68.24; H, 3.82; S, 16.07.

1,1,2-Trichloro-4,4-bis(n-ethylthio)-1,3-butadiene (2d). Yield 41\%; Oil, $\mathrm{R}_{f}$ (Hexane): 0.70; IR (film, $\left.\mathrm{cm}^{-1}\right): 1546(\mathrm{C}=\mathrm{C}), 2870,2927,2971(\mathrm{C}-\mathrm{H}) .{ }^{1} \mathrm{H}$ NMR $\left(499.83 \mathrm{MHz}, \mathrm{CDCl}_{3}\right): \delta 6.18(\mathrm{~s}, 1 \mathrm{H}$, vinyl-H), 2.74-2.82 (m, $\left.J=7.32 \mathrm{~Hz}, 4 \mathrm{H}, \mathrm{S}-\mathrm{CH}_{2}\right), 1.19-1.25\left(\mathrm{~m}, J=7.32 \mathrm{~Hz}, 6 \mathrm{H}, \mathrm{CH}_{3}\right) ;{ }^{13} \mathrm{C}$ NMR $\left(125.68 \mathrm{MHz}, \mathrm{CDCl}_{3}\right): \delta 119.28,121.53,124.98,142.34\left(\mathrm{C}_{\text {butad }}\right), 12.86,13.98\left(\mathrm{CH}_{3}\right)$, 26.93, $27.03\left(\mathrm{CH}_{2}\right)$; MS (+ESI): $\mathrm{m} / z$ 279.41 [M+H] ${ }^{+} ; \mathrm{C}_{8} \mathrm{H}_{11} \mathrm{Cl}_{3} \mathrm{~S}_{2}$ (M, 277.66); Calcd. C, 34.61; H, 3.99; S, 23.10. Found C, 34.92; H, 4.02; S, 23.65.

1,1,2,4-Tetrakis( $n$-ethylthio)-1-buten-3-yne (6d). Yield 27\%; Oil, $\mathrm{R}_{f}$ (Hexane): 0.35; IR (film, $\left.\mathrm{cm}^{-1}\right): 1531(\mathrm{C}=\mathrm{C}), 2144(\mathrm{C} \equiv \mathrm{C}), 2872,2930,2958(\mathrm{C}-\mathrm{H}) .{ }^{1} \mathrm{H}$ NMR $\left(499.83 \mathrm{MHz}, \mathrm{CDCl}_{3}\right): \delta$ 2.62-2.90 (m, $\left.J=7.32 \mathrm{~Hz}, 8 \mathrm{H}, \mathrm{S}-\mathrm{CH}_{2}\right), 1.18-1.47\left(\mathrm{~m}, J=7.32 \mathrm{~Hz}, 12 \mathrm{H}, \mathrm{CH}_{3}\right) ;{ }^{13} \mathrm{C} \mathrm{NMR}(125.68$ $\left.\mathrm{MHz}, \mathrm{CDCl}_{3}\right): \delta 87.59,89.40,124.60,139.82\left(\mathrm{C}_{\text {butenyne }}\right) ; 27.59,27.18,27.03,26.92\left(\mathrm{CH}_{2}\right) ; 13.95$, 
13.93, 13.62, $13.13\left(\mathrm{CH}_{3}\right)$; MS (+ESI): $\mathrm{m} / z$ 292.94 [M] ${ }^{+} ; \mathrm{C}_{12} \mathrm{H}_{20} \mathrm{~S}_{4}$ (M, 292.55); Calcd. C, 49.27; H, 6.89; S, 43.84. Found 49.85; H, 6.02; S, 44.05.

\section{General procedure 2}

2H-pentachlorobutadiene 1 ( $1 \mathrm{~g}, 4.4 \mathrm{mmol}$ ) or 2H-1,1,3,4-tetrachloro-4-bromobutadiene 9 (1 g, $3.7 \mathrm{mmol}$ ) and thiols (4.4 mmol for the reaction with 1, $3.7 \mathrm{mmol}$ for the reaction with 9) were stirred in a mixture of EtOH $(30 \mathrm{~mL})$ and aqueous solution of $\mathrm{NaOH}(1.2 \mathrm{~g}$ and $8 \mathrm{ml}$ water) for $30 \mathrm{~min}$. at room temperature. Ether was added the reaction mixture. The organic layer was separated and washed with water $(4 \times 30 \mathrm{~mL})$, and dried with $\mathrm{MgSO}_{4}$. The solvent was evaporated and the products were purified by column chromatography over silica gel (petroleum ether).

1,1,2-Trichloro-4-(4-bromophenylthio)-1-buten-3-yne (7a). Yield 65\%; White solid, mp: 68$69{ }^{\circ} \mathrm{C} . \mathrm{R}_{f}$ (Petroleum ether): 0.67; IR $\left(\mathrm{KBr}, \mathrm{cm}^{-1}\right): 2157(\mathrm{C} \equiv \mathrm{C}), 3025\left(=\mathrm{C}-\mathrm{H}_{\text {arom }}\right) ; \mathrm{UV}-\mathrm{vis}\left(\mathrm{CHCl}_{3}\right)$ : $\lambda_{\max }(\log \varepsilon)=266 \mathrm{~nm}(4.0) ;{ }^{1} \mathrm{H}$ NMR $\left(499.83 \mathrm{MHz}, \mathrm{CDCl}_{3}\right): \delta 7.20-7.45\left(\mathrm{~m}, 4 \mathrm{H}, \mathrm{H}_{\text {arom }}\right) ;{ }^{13} \mathrm{C}$ NMR (125.68 MHz, $\left.\mathrm{CDCl}_{3}\right): \delta 128.50,128.66,132.67,132.87\left(\mathrm{CH}_{\text {arom }}\right), 87.78,91.03,112.85$,

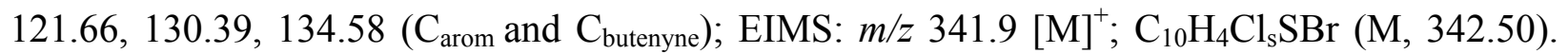
Calcd. C, 35.07; H, 1.17; S, 9.36. Found C, 35.67; H, 1.12; S, 9.25.

1,1,2-Trichloro-4-(2-naphtylthio)-1-buten-3-yne (6c). Yield 43\%; White solid, mp: 71-73 ${ }^{\circ} \mathrm{C}$. $\mathrm{R}_{f}$ (Petroleum ether): 0.71; IR ( $\left.\mathrm{KBr}, \mathrm{cm}^{-1}\right): 2149(\mathrm{C} \equiv \mathrm{C}), 3032\left(=\mathrm{C}-\mathrm{H}_{\text {arom }}\right) ; \mathrm{UV}$-vis $\left(\mathrm{CHCl}_{3}\right): \lambda_{\max }$ $(\log \varepsilon)=259 \mathrm{~nm}(5.2) ;{ }^{1} \mathrm{H}$ NMR $\left(499.83 \mathrm{MHz}, \mathrm{CDCl}_{3}\right): \delta 7.39-7.85\left(\mathrm{~m}, 7 \mathrm{H}, \mathrm{H}_{\text {arom }}\right) ;{ }^{13} \mathrm{C}$ NMR $\left(125.68 \mathrm{MHz}, \mathrm{CDCl}_{3}\right): \delta 88.88,90.67,113.04,124.64,125.94,126.74,127.36,127.52,128.00$, $128.13,128.26,129.63,132.62,133.97\left(\mathrm{C}_{\text {arom }}, \mathrm{CH}_{\text {arom }}, \mathrm{C}_{\text {butenyne }}\right) ; \mathrm{C}_{14} \mathrm{H}_{7} \mathrm{Cl}_{3} \mathrm{~S}(\mathrm{M}, 313,5)$. Calcd. C, 53.20; H, 2.85; S, 10.40. Found C, 53.68; H, 2.38; S, 9.63.

1,1,2-Trichloro-4-(1-octylthio)-1-buten-3-yne (7e). Yield 52\%; Oil, $\mathrm{R}_{f}$ (Petroleum ether): 0.87; IR (film, $\left.\mathrm{cm}^{-1}\right): 2152(\mathrm{C} \equiv \mathrm{C}), 2855,2925(\mathrm{C}-\mathrm{H}) . \mathrm{UV}$-vis $\left(\mathrm{CHCl}_{3}\right): \lambda_{\max }(\log \varepsilon)=244 \mathrm{~nm}(4.6) ;{ }^{1} \mathrm{H}$ NMR (499.83 MHz, $\left.\mathrm{CDCl}_{3}\right): \delta 0.8\left(\mathrm{t}, J=7.32 \mathrm{~Hz}, 3 \mathrm{H}, \mathrm{CH}_{3}\right), 1.2-1.4\left(\mathrm{~m}, 10 \mathrm{H},-\left(\mathrm{CH}_{2}\right)_{5^{-}}\right), 1.70$ $\left(\mathrm{m}, 2 \mathrm{H}, \mathrm{S}-\mathrm{CH}_{2}-\mathrm{CH}_{2}\right), 2.75\left(\mathrm{t}, J=7.32,2 \mathrm{H}, \mathrm{S}-\mathrm{CH}_{2}\right){ }^{13} \mathrm{C} \mathrm{NMR}\left(125.68 \mathrm{MHz}, \mathrm{CDCl}_{3}\right): \delta 13.04$ $\left(\mathrm{CH}_{3}\right)$; 21.62, 27.14, 27.99, 28.08, 28.33, 30.75, $35.09\left(\mathrm{CH}_{2}\right) ; 85.08,92.04,112.01,123.88$

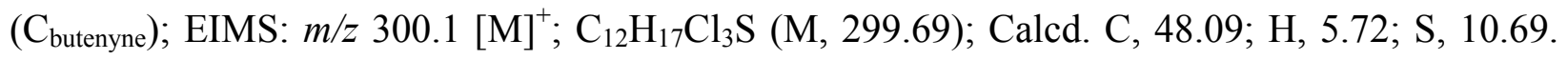
Found. C, 48.31; H, 5.82; S 10.44.

1,1,2-Trichloro-4-(1-decylthio)-1-buten-3-yne (7f). Yield 41\%; Oil, $\mathrm{R}_{f}$ (Petroleum ether): 0.75; IR (film, $\left.\mathrm{cm}^{-1}\right): 2147(\mathrm{C} \equiv \mathrm{C}), 2855,2925(\mathrm{C}-\mathrm{H}) . \mathrm{UV}$-vis $\left(\mathrm{CHCl}_{3}\right): \lambda_{\max }(\log \varepsilon)=244 \mathrm{~nm}(4.1) ;{ }^{1} \mathrm{H}$ NMR (499.83 MHz, $\left.\mathrm{CDCl}_{3}\right): \delta 0.81\left(\mathrm{t}, J=7.32 \mathrm{~Hz}, 3 \mathrm{H}, \mathrm{CH}_{3}\right), 1.2-1.4\left(\mathrm{~m}, 14 \mathrm{H},-\left(\mathrm{CH}_{2}\right)_{7^{-}}\right), 1.69$ $\left(\mathrm{m}, 2 \mathrm{H}, \mathrm{S}-\mathrm{CH}_{2}-\mathrm{CH}_{2}\right), 2.73\left(\mathrm{t}, J=7.32,2 \mathrm{H}, \mathrm{S}-\mathrm{CH}_{2}\right){ }^{13} \mathrm{C} \mathrm{NMR}\left(125.68 \mathrm{MHz}, \mathrm{CDCl}_{3}\right): \delta 13.09$ $\left(\mathrm{CH}_{3}\right)$; 21.67, 27.15, 28.05, 28.09, 28.29, 28.44, 28.52, 30.89, $35.09\left(\mathrm{CH}_{2}\right)$; 85.10, 92.07, 112.03,

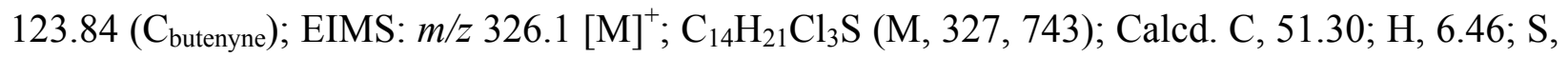
9.78. Found C, 51.37; H, 6.81; S, 9.93.

1,1,2-Trichloro-4-(1-dodecylthio)-1-buten-3-yne (7g). Yield 48\%; Oil, $\mathrm{R}_{f}$ (Petroleum ether): 0.85; IR (film, cm $\left.{ }^{-1}\right): 2152(\mathrm{C} \equiv \mathrm{C}), 2854,2925(\mathrm{C}-\mathrm{H}) . \mathrm{UV}-\mathrm{vis}\left(\mathrm{CHCl}_{3}\right): \lambda_{\max }(\log \varepsilon)=244 \mathrm{~nm}(5.1)$; ${ }^{1} \mathrm{H}$ NMR (499.83 MHz, $\left.\mathrm{CDCl}_{3}\right): \delta 0.79\left(\mathrm{t}, J=7.32 \mathrm{~Hz}, 3 \mathrm{H}, \mathrm{CH}_{3}\right), 1.2-1.4\left(\mathrm{~m}, 18 \mathrm{H},-\left(\mathrm{CH}_{2}\right)_{9}-\right), 1.69$ $\left(\mathrm{m}, 2 \mathrm{H}, \mathrm{S}-\mathrm{CH}_{2}-\mathrm{CH}_{2}\right), 2.75\left(\mathrm{t}, J=7.32,2 \mathrm{H}, \mathrm{S}-\mathrm{CH}_{2}\right){ }^{13} \mathrm{C} \mathrm{NMR}\left(125.68 \mathrm{MHz}, \mathrm{CDCl}_{3}\right): \delta 13.07$ 
$\left(\mathrm{CH}_{3}\right) ; 21.67,27.14,28.03,28.32,28.34,28.42,28.54,28.60,28.62,30.90,35.10\left(\mathrm{CH}_{2}\right) ; 85.10$,

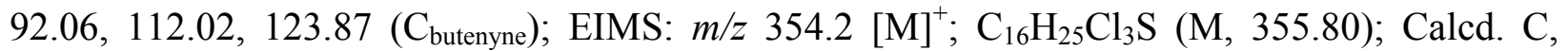
54.01; H, 7.08; S, 9.01. Found C, 54.14; H, 7.22; S, 9.49.

1,1,2-Trichloro-4-(1-hexadecylthio)-1-buten-3-yne (7h). Yield 32\%; Oil, $\mathrm{R}_{f}$ (Petroleum ether); 0.86; IR (film, $\left.\mathrm{cm}^{-1}\right): 2152(\mathrm{C} \equiv \mathrm{C}), 2855,2925(\mathrm{C}-\mathrm{H})$. UV-vis $\left(\mathrm{CHCl}_{3}\right): \lambda_{\max }(\log \varepsilon)=243 \mathrm{~nm}(4.1)$; ${ }^{1} \mathrm{H}$ NMR $\left(499.83 \mathrm{MHz}, \mathrm{CDCl}_{3}\right): \delta 0.88\left(\mathrm{t}, J=7.32 \mathrm{~Hz}, 3 \mathrm{H}, \mathrm{CH}_{3}\right), 1.2-1.4\left(\mathrm{~m}, 26 \mathrm{H},-\left(\mathrm{CH}_{2}\right)_{13^{-}}\right)$, $1.76\left(\mathrm{~m}, 2 \mathrm{H}, \mathrm{S}-\mathrm{CH}_{2}-\mathrm{CH}_{2}\right), 2.80\left(\mathrm{t}, J=7.32,2 \mathrm{H}, \mathrm{S}_{-} \mathrm{CH}_{2}\right){ }^{13} \mathrm{C} \mathrm{NMR}\left(125.68 \mathrm{MHz}, \mathrm{CDCl}_{3}\right): \delta$ $13.09\left(\mathrm{CH}_{3}\right) ; 21.69,27.15,28.05,28.08,28.35,28.36,28.44,28.47,28.56,28.64,28.67,28.69$, 28.71, 30.93, $35.10\left(\mathrm{CH}_{2}\right) ; 85.11,92.07,112.04,123.86$ ( $\left.\mathrm{C}_{\text {butenyne }}\right) ; \mathrm{C}_{20} \mathrm{H}_{33} \mathrm{Cl}_{3} \mathrm{~S}(\mathrm{M}, 411.50)$ Calcld. C, 58.30; H, 8.02; S, 7.77. Found. C, 58.25; H, 7.85; S, 7.55.

1,1,2-Trichloro-4-(1-octadecylthio)-1-buten-3-yne (7i). Yield 23\%; Oil, $\mathrm{R}_{f}$ (Petroleum ether); 0.82; IR (film, $\left.\mathrm{cm}^{-1}\right): 2157(\mathrm{C} \equiv \mathrm{C}), 2855,2925(\mathrm{C}-\mathrm{H})$. UV-vis $\left(\mathrm{CHCl}_{3}\right): \lambda_{\max }(\log \varepsilon)=243 \mathrm{~nm}(4.5)$; ${ }^{1} \mathrm{H}$ NMR $\left(499.83 \mathrm{MHz}, \mathrm{CDCl}_{3}\right): \delta 0.86\left(\mathrm{t}, J=7.32 \mathrm{~Hz}, 3 \mathrm{H}, \mathrm{CH}_{3}\right), 1.2-1.4\left(\mathrm{~m}, 30 \mathrm{H},-\left(\mathrm{CH}_{2}\right)_{15^{-}}\right)$, $1.76\left(\mathrm{~m}, 2 \mathrm{H}, \mathrm{S}-\mathrm{CH}_{2}-\mathrm{CH}_{2}\right), 2.80\left(\mathrm{t}, J=7.32,2 \mathrm{H}, \mathrm{S}-\mathrm{CH}_{2}\right){ }^{13} \mathrm{C} \mathrm{NMR}\left(125.68 \mathrm{MHz}, \mathrm{CDCl}_{3}\right): \delta$ $13.09\left(\mathrm{CH}_{3}\right) ; 21.69,27.15,27.54,28.05,28.09,28.35,28.37,28.44,28.47,28.56,28.59,28.60$, 28.64, 28.67, 28.71, 30.94, $35.10\left(\mathrm{CH}_{2}\right) ; 85.11,92.06,112.03,123.85$ ( $\left.\mathrm{C}_{\text {butenyne }}\right) ; \mathrm{C}_{22} \mathrm{H}_{37} \mathrm{Cl}_{3} \mathrm{~S}(\mathrm{M}$, 439.96) Calcd. C, 60.06; H, 8.47; S, 7.28. Found C, 60.61; H,8.49; S, 7.29.

1-Bromo-1,2-dichloro-4-(1-octylthio)-1-buten-3-yne (10e). Yield 67\%; Oil, R (Petroleum ether): 0.85; IR(film, cm $\left.{ }^{-1}\right): 2146(\mathrm{C} \equiv \mathrm{C}), 2855,2956(\mathrm{C}-\mathrm{H}) . \mathrm{UV}-\mathrm{vis}\left(\mathrm{CHCl}_{3}\right): \lambda_{\max }(\log \varepsilon)=242$ $\mathrm{nm}(4.5) ;{ }^{1} \mathrm{H}$ NMR $\left(499.83 \mathrm{MHz}, \mathrm{CDCl}_{3}\right): \delta 0.82\left(\mathrm{t}, J=7.32 \mathrm{~Hz}, 3 \mathrm{H}, \mathrm{CH}_{3}\right), 1.22-1.47(\mathrm{~m}, 10 \mathrm{H},-$ $\left.\left.\left(\mathrm{CH}_{2}\right)_{5}\right)^{-}\right) 1.70\left(\mathrm{~m}, 2 \mathrm{H}, \mathrm{S}-\mathrm{CH}_{2}-\mathrm{CH}_{2}\right), 2.74\left(\mathrm{t}, J=7.32,2 \mathrm{H}, \mathrm{S}_{-} \mathrm{CH}_{2}\right){ }^{13} \mathrm{C}$ NMR $(125.68 \mathrm{MHz}$, $\left.\mathrm{CDCl}_{3}\right): \delta 13.04\left(\mathrm{CH}_{3}\right) ; 21.62,27.14,28.00,28.08,28.09,30.75,35.22\left(\mathrm{CH}_{2}\right) ; 86,70,91.37$, 112.55, 123.27 ( $\left.\mathrm{C}_{\text {butenyne }}\right) ; \mathrm{C}_{12} \mathrm{H}_{17} \mathrm{SCl}_{2} \mathrm{Br}$ (M, 344.14); Calcld. C, 41.88; H, 4.98; S, 9.32. Found. C, 41.31; H, 5.02; S 9.63.

1-Bromo-1,2-dichloro-4-(1-decylthio)-1-buten-3-yne (10f). Yield 60\%; Oil, $\mathrm{R}_{f}$ (Petroleum ether): 0.77; IR(film, $\left.\mathrm{cm}^{-1}\right): 2146(\mathrm{C} \equiv \mathrm{C}), 2855,2925(\mathrm{C}-\mathrm{H}) . \mathrm{UV}-\mathrm{vis}\left(\mathrm{CHCl}_{3}\right): \lambda_{\max }(\log \varepsilon)=242$ $\mathrm{nm}(4.0) ;{ }^{1} \mathrm{H}$ NMR $\left(499.83 \mathrm{MHz}, \mathrm{CDCl}_{3}\right): \delta 0.81\left(\mathrm{t}, J=7.32 \mathrm{~Hz}, 3 \mathrm{H}, \mathrm{CH}_{3}\right), 1.2-1.4(\mathrm{~m}, 14 \mathrm{H},-$ $\left.\left(\mathrm{CH}_{2}\right)_{7^{-}}\right), 1.70\left(\mathrm{~m}, 2 \mathrm{H}, \mathrm{S}-\mathrm{CH}_{2}-\mathrm{CH}_{2}\right), 2.74\left(\mathrm{t}, J=7.32,2 \mathrm{H}, \mathrm{S}-\mathrm{CH}_{2}\right){ }^{13} \mathrm{C}$ NMR $(125.68 \mathrm{MHz}$, $\left.\mathrm{CDCl}_{3}\right): \delta 13.07\left(\mathrm{CH}_{3}\right) ; 21.65,27.13,28.04,28.27,28.42,28.43,28.49,30.87,35.21\left(\mathrm{CH}_{2}\right)$; 86.69, 92.23, 113.84, 123.27 ( $\mathrm{C}_{\text {butenyne }}$ ); $\mathrm{C}_{14} \mathrm{H}_{21} \mathrm{Cl}_{2} \mathrm{BrS}$ (M, 372.19) Calcd. C, 45.18; H, 5.68; $\mathrm{S}$, 8.61. Found C, 45.22; H, 5.42; S 8.42.

1-Bromo-1,2-dichloro-4-(1-dodecylthio)-1-buten-3-yne (10g). Yield 63\%; Oil, $\mathrm{R}_{f}$ (Petroleum ether): 0.82; IR (film, $\left.\mathrm{cm}^{-1}\right): 2147(\mathrm{C} \equiv \mathrm{C}), 2853,2924(\mathrm{C}-\mathrm{H}) . \mathrm{UV}-\mathrm{vis}\left(\mathrm{CHCl}_{3}\right): \lambda_{\max }(\log \varepsilon)=243$ $\mathrm{nm}(5.1) ;{ }^{1} \mathrm{H}$ NMR $\left(499.83 \mathrm{MHz}, \mathrm{CDCl}_{3}\right): \delta 0.81\left(\mathrm{t}, J=7.32 \mathrm{~Hz}, 3 \mathrm{H}, \mathrm{CH}_{3}\right), 1.19-1.35(\mathrm{~m}, 18 \mathrm{H},-$ $\left.\left(\mathrm{CH}_{2}\right)_{9-}\right), 1.71\left(\mathrm{~m}, 2 \mathrm{H}, \mathrm{S}-\mathrm{CH}_{2}-\mathrm{CH}_{2}\right), 2.74\left(\mathrm{t}, J=7.32,2 \mathrm{H}, \mathrm{S}_{-} \mathrm{CH}_{2}\right){ }^{13} \mathrm{C} \mathrm{NMR}(125.68 \mathrm{MHz}$, $\left.\mathrm{CDCl}_{3}\right): \delta 13.08\left(\mathrm{CH}_{3}\right) ; 21.67,27.14,28.05,28.33,28.40,28.43,28.55,28.61,28.62,30.91$, $35.04\left(\mathrm{CH}_{2}\right) ; 86.70,91.38,112.54,122.68$ ( $\left.\mathrm{C}_{\text {butenyne }}\right) ; \mathrm{C}_{16} \mathrm{H}_{25} \mathrm{Cl}_{2} \mathrm{BrS}$ (M, 400.25); Calcd. C, 48.01; H, 6.29; S, 8.01. Found C, 47.96; H, 6.18; S, 8.27.

1-Bromo-1,2-dichloro-4-(1-hexadecylthio)-1-buten-3-yne (10h). Yield 50\%; Oil, $\mathrm{R}_{f}$ (Petroleum ether); 0.83; IR (film, $\left.\mathrm{cm}^{-1}\right): 2146(\mathrm{C} \equiv \mathrm{C}), 2851,2921(\mathrm{C}-\mathrm{H})$. UV-vis $\left(\mathrm{CHCl}_{3}\right): \lambda_{\max }$ 
$(\log \varepsilon)=244 \mathrm{~nm}(4.1) ;{ }^{1} \mathrm{H}$ NMR $\left(499.83 \mathrm{MHz}, \mathrm{CDCl}_{3}\right): \delta 0.81\left(\mathrm{t}, J=7.32 \mathrm{~Hz}, 3 \mathrm{H}, \mathrm{CH}_{3}\right), 1.2-1.4$ (m, 26H, - $\left.\left(\mathrm{CH}_{2}\right)_{13^{-}}\right), 1.71\left(\mathrm{~m}, 2 \mathrm{H}, \mathrm{S}-\mathrm{CH}_{2}-\mathrm{CH}_{2}\right), 2.73\left(\mathrm{t}, J=7.32,2 \mathrm{H}, \mathrm{S}-\mathrm{CH}_{2}\right){ }^{13} \mathrm{C}$ NMR $(125.68$ $\left.\mathrm{MHz}, \mathrm{CDCl}_{3}\right): \delta 13.08\left(\mathrm{CH}_{3}\right) ; 21.67,27.13,27.15,28.04,28.35,28.40,28.42,28.44,28.54$, 28.62, 28.65, 28.67, 28.68, 30.92, $35.03\left(\mathrm{CH}_{2}\right) ; 86.70,92.24,112.54,124.10$ (C butenyne); MS (+APCI): $m / z 455.93[\mathrm{M}]^{+} ; \mathrm{C}_{20} \mathrm{H}_{33} \mathrm{Cl}_{2} \mathrm{BrS}$ (M, 456.36); Calcd. C, 52.64; H, 7.29; S, 7.02. Found C, 52.69; H, 7.69; S, 7.58.

1-Bromo-1,2-dichloro-4-(1-octadecylthio)-1-buten-3-yne (10i). Yield 44\%; Oil, $\mathrm{R}_{f}$ (Petroleum ether); 0.79; IR (film, cm $\left.{ }^{-1}\right): 2148(\mathrm{C} \equiv \mathrm{C}), 2853,2923(\mathrm{C}-\mathrm{H})$. UV-vis $\left(\mathrm{CHCl}_{3}\right): \lambda_{\max }(\log \varepsilon)=244$ nm (3.9); ${ }^{1} \mathrm{H}$ NMR $\left(499.83 \mathrm{MHz}, \mathrm{CDCl}_{3}\right): \delta 0.81\left(\mathrm{t}, J=7.32 \mathrm{~Hz}, 3 \mathrm{H}, \mathrm{CH}_{3}\right), 1.2-1.4(\mathrm{~m}, 30 \mathrm{H},-$ $\left.\left(\mathrm{CH}_{2}\right)_{15^{-}}\right), 1.71\left(\mathrm{~m}, 2 \mathrm{H}, \mathrm{S}-\mathrm{CH}_{2}-\mathrm{CH}_{2}\right), 2.74\left(\mathrm{t}, J=7.32,2 \mathrm{H}, \mathrm{S}-\mathrm{CH}_{2}\right){ }^{13} \mathrm{C}$ NMR $(125.68 \mathrm{MHz}$, $\left.\mathrm{CDCl}_{3}\right): \delta 13.09\left(\mathrm{CH}_{3}\right) 21.68,27.13,27.15,28.04,28.24,28.33,28.35,28.39,228.42,28.44$, 28.55, 28.63, 28.65, 28.67, 28.69, 30.92, $35.07\left(\mathrm{CH}_{2}\right) ; 86.69,91.36,110.60,125.47$ ( $\left.\mathrm{C}_{\text {butenyne }}\right)$; $\mathrm{C}_{22} \mathrm{H}_{37} \mathrm{Cl}_{2} \mathrm{BrS}$ (M, 484.41); Calcd. C, 54.55; H, 7.69; S, 6.62. Found C, 54.78; H, 7.67; S, 6.04.

\section{General procedure 3}

Tris- or tetrakis-thiosubstituted butadiene compound $(0.9 \mathrm{mmol})$ in $50 \mathrm{~mL}$ Petroleum ether (30$\left.50{ }^{\circ} \mathrm{C}\right)$ was mixed with potassium tert-butoxide $(0.2 \mathrm{~g}, 1.8 \mathrm{mmol})$ for $4 \mathrm{~h}$ at room temperature. For 12-13c, Tetrahydofuran was used as solvent instead of Petroleum ether. Ether and water added to the reaction mixture and then organic layer was separated, dried with anhydrous $\mathrm{MgSO}_{4}$. The solvent was evaporated and the residue was purified by column chromatography over silica gel (petroleum ether/chloroform).

1-Chloro-1,4,4-tris(4-bromophenylthio)-1,2,3-butatriene (12a) and 2-Chloro-1,1,4-tris(4bromophenylthio)-1-buten-3-yne (13a). Yield 82\%; Bright yellow crystalline solid, mp: 119$121^{\circ} \mathrm{C} . \mathrm{R}_{f}$ (Petroleum ether): 0.35; IR $\left(\mathrm{KBr}, \mathrm{cm}^{-1}\right): 2037,859(\mathrm{C}=\mathrm{C}=\mathrm{C}=\mathrm{C}), 2142(\mathrm{C} \equiv \mathrm{C}), 1576$ $(\mathrm{C}=\mathrm{C}), 3073$ (=C-Harom). UV-vis $\left(\mathrm{CHCl}_{3}\right): \lambda_{\max }(\log \varepsilon)=384 \mathrm{~nm}(4.9) ;{ }^{1} \mathrm{H}$ NMR $(499.83 \mathrm{MHz}$, $\left.\mathrm{CDCl}_{3}\right): \delta$ 7.12-7.44 (m, 12H, $\left.\mathrm{H}_{\text {arom }}\right){ }^{13} \mathrm{C}$ NMR $\left(125.68 \mathrm{MHz}, \mathrm{CDCl}_{3}\right): \delta 86.24,93.19,101.80$, $115.53,120.08,120.76,122.19,122.49,123.25,127.17,128.47,128.97,129.15,129.81,130.07$, 130.22, 130.57, 130.83, 130.97, 131.23, 131.31, 131.45, 131.46, 131.47, 131.60, 132.19, 133.00, 134.23, 134.52, 137.53, 145.88, $153.13\left(\mathrm{C}_{\text {arom }}, \mathrm{CH}_{\text {arom }}, \mathrm{C}_{\text {butatriene, }} \mathrm{C}_{\text {butenyne }}\right)$; $\mathrm{MS}(+\mathrm{ESI}): \mathrm{m} / \mathrm{z}$ $648.84[\mathrm{M}+\mathrm{H}]^{+} ; \mathrm{C}_{22} \mathrm{H}_{12} \mathrm{ClBr}_{3} \mathrm{~S}_{3}(\mathrm{M}, 647.50)$; Calcd. C, 40.80; H, 1.85; S, 14.83. Found C, 40.67; $\mathrm{H}, 1.23 ; \mathrm{S}, 15.03$.

1-Chloro-1,4,4-tris(4-fluorophenylthio)-1,2,3-butatriene (12b) and 2-Chloro-1,1,4-tris(4fluorophenylthio)-1-buten-3-yne (13b). Yield 76\%; Oil, $\mathrm{R}_{f}$ [Petroleum ether/ $\left.\mathrm{CHCl}_{3}(1: 1)\right]$ : 0.40; IR (film, cm $\left.{ }^{-1}\right)$ : 2043, $870(\mathrm{C}=\mathrm{C}=\mathrm{C}=\mathrm{C}), 2143(\mathrm{C} \equiv \mathrm{C}), 1589(\mathrm{C}=\mathrm{C}), 3068\left(=\mathrm{C}-\mathrm{H}_{\text {arom }}\right) . \mathrm{UV}-$ $\operatorname{vis}\left(\mathrm{CHCl}_{3}\right): \lambda_{\max }(\log \varepsilon)=316 \mathrm{~nm}(5.0) ;{ }^{1} \mathrm{H} \mathrm{NMR}\left(499.83 \mathrm{MHz}, \mathrm{CDCl}_{3}\right): \delta$ 7.12-7.51 (m, $12 \mathrm{H}$, $\mathrm{H}_{\text {arom }}{ }^{13} \mathrm{C}$ NMR $\left(125.68 \mathrm{MHz}, \mathrm{CDCl}_{3}\right): \delta 86.07,93.75,110.00,114.69,115.93,116.11,116.29$, $116.57,116.75,116.82,126.95,127.49,128.53,129.10,129.16,130.94,132.51,132.57,133.90$, 133.97, 135.12, 135.92, 135.99, 136.58, 140.26, 143.03, 161.64, 162.35, 163.37, 163.62, 164.33, $164.43\left(\mathrm{C}_{\text {arom }}, \mathrm{CH}_{\text {arom }}, \mathrm{C}_{\text {butatriene, }} \mathrm{C}_{\text {butenyne }}\right) ; \mathrm{MS}(+\mathrm{ESI}): \mathrm{m} / \mathrm{z} 465.13[\mathrm{M}]^{+} ; \mathrm{C}_{22} \mathrm{H}_{12} \mathrm{ClF}_{3} \mathrm{~S}_{3}(\mathrm{M}$, 464.97); Calcd. C, 56.83; H, 2.60; S, 20.69. Found C, 57.0; H, 2.93; S, 21.07. 
1-Chloro-1,4,4-tris(2-naphtylthio)-1,2,3-butatriene (12c) and 2-Chloro-1,1,4-tris(2naphtylthio)-1-buten-3-yne (13c). Yield 67\%; Yellow crystalline solid, mp: $185-187{ }^{\circ} \mathrm{C} . \mathrm{R}_{f}$ [Petroleum ether/ $\mathrm{CHCl}_{3}(1: 1)$ ]: 0.30; IR $\left(\mathrm{KBr}, \mathrm{cm}^{-1}\right): 2043,856(\mathrm{C}=\mathrm{C}=\mathrm{C}=\mathrm{C}), 2141(\mathrm{C} \equiv \mathrm{C}), 1588$ $(\mathrm{C}=\mathrm{C}), 3055$ (=C-H $\left.\mathrm{H}_{\text {arom }}\right)$. UV-vis $\left(\mathrm{CHCl}_{3}\right): \lambda_{\max }(\log \varepsilon)=392 \mathrm{~nm}(4.2) ;{ }^{1} \mathrm{H}$ NMR $(499.83 \mathrm{MHz}$, $\left.\mathrm{CDCl}_{3}\right): \delta 7.29-7.85\left(\mathrm{~m}, 21 \mathrm{H}, \mathrm{H}_{\text {arom }}\right){ }^{13} \mathrm{C} \mathrm{NMR}\left(125.68 \mathrm{MHz}, \mathrm{CDCl}_{3}\right): \delta 84.02,94.63,115.05$, $124.39,125.35,126.34,126.55,126.60,126.69,126.72,126.76,126.78,126.83,126.86,126.91$, $126.93,127.04,127.06,127.07,127.14,127.42$, 127.63, 127.78, 127.80, 127.87, 127.89, 127.90, 127.92 , 127.97, 128.03, 128.32, 128.57, 128.62, 128.99, 129.01, 129.08, 129.20, 129.28, 129.64, $129.72,129.86,129.90,129.91,130.06,131.14,132.06,132.26,132.46,133.16,134.30\left(\mathrm{C}_{\text {arom, }}\right.$ $\mathrm{CH}_{\text {arom}}, \mathrm{C}_{\text {butatriene, }} \mathrm{C}_{\text {butenyne}}$ ); $\mathrm{MS}$ (+APCI): $\mathrm{m} / z 561.31[\mathrm{M}]^{+} ; \mathrm{C}_{43} \mathrm{H}_{21} \mathrm{ClS}_{3}(\mathrm{M}, 561.18)$; Calcd. C, 72.77; H, 3.77; S, 17.14. Found C, 73.21; H, 3.93; S, 17.07.

1,1,4,4-Tetrakis(4-bromophenylthio)butatriene (14a). Yield 94\%; Bright yellow crystalline solid, mp: $179-181^{\circ} \mathrm{C} . \mathrm{R}_{f}$ [Petroleum ether $/ \mathrm{CCl}_{4}(1: 1)$ ]: 0.42; IR (KBr): $867(\mathrm{C}=\mathrm{C}=\mathrm{C}=\mathrm{C}), 3048$ $\left(=\mathrm{C}-\mathrm{H}_{\text {arom }}\right) .{ }^{1} \mathrm{H}$ NMR $\left(499.83 \mathrm{MHz}, \mathrm{CDCl}_{3}\right): \delta 7.09$ (d, 8H, $\left.\mathrm{H}_{\text {arom }}\right), 7.31\left(\mathrm{~d}, 8 \mathrm{H}, \mathrm{H}_{\text {arom }}\right) ;{ }^{13} \mathrm{C}$ NMR $\left(125.68 \mathrm{MHz}, \mathrm{CDCl}_{3}\right): \delta 109.76,122.07,130.70,131.30,132.69$ and $149.91\left(\mathrm{C}_{\text {arom, }} \mathrm{CH}_{\text {arom }}\right.$ and $\mathrm{C}_{\text {butatriene }}$ ); MS (+ESI): $m / z$ 800.11 [M] ${ }^{+} ; \mathrm{C}_{28} \mathrm{H}_{16} \mathrm{Br}_{4} \mathrm{~S}_{4}(\mathrm{M}, 800.30)$. Calcd. C, 40.80; H, 1.85; S, 14.83. Found C, 40.67; H, 1.23; S, 15.03.

\section{General procedure 4}

Monothiosubstituted butenyne compound $(1.5 \mathrm{mmol})$ and bromine $(0.25 \mathrm{~g}, 1.5 \mathrm{mmol})$ were stirred in $\mathrm{CCl}_{4}(30 \mathrm{~mL})$ for $3 \mathrm{~h}$ at room temperature. Ether was added to the reaction mixture and extracted with $100 \mathrm{ml}$ of $3 \%$ aqueous solution of $\mathrm{Na}_{2} \mathrm{~S}_{2} \mathrm{O}_{5}$ twice. The organic layer was separated, washed with water $(4 \times 30 \mathrm{~mL})$, dried with anhydrous $\mathrm{MgSO}_{4}$. The solvent was evaporated and the residue was purified by column chromatography over silica gel (petroleum ether).

1,2-Dibromo-3,4,4-trichloro-1-(4-bromophenylthio)-1,3-butadiene 8a. Yield 82\%; White solid, mp: $60-62{ }^{\circ} \mathrm{C} . \mathrm{R}_{f}$ (Petroleum ether): 0.67; IR (film, $\left.\mathrm{cm}^{-1}\right): 1599,1564(\mathrm{C}=\mathrm{C}), 3080$ (=C$\left.\mathrm{H}_{\text {arom }}\right)$, UV-vis $\left(\mathrm{CHCl}_{3}\right): \lambda_{\max }(\log \varepsilon)=243(4.02) ;{ }^{1} \mathrm{H} \mathrm{NMR}\left(499.83 \mathrm{MHz}, \mathrm{CDCl}_{3}\right): \delta 7.18-7.48(\mathrm{~m}$, $\left.4 \mathrm{H}, \mathrm{H}_{\text {arom }}\right) ;{ }^{13} \mathrm{C} \mathrm{NMR}\left(125.68 \mathrm{MHz}, \mathrm{CDCl}_{3}\right): \delta 111.62,119.87,121.83,123.36,129.67,131.57$, 131.78, 132.88, 134.79; $\mathrm{C}_{10} \mathrm{H}_{4} \mathrm{Br}_{3} \mathrm{Cl}_{3} \mathrm{~S}$ (M, 502, 27) Calcd. C, 23.91; H, 0.80; S, 6.38. Found C, $24.51 ; \mathrm{H}, 0.86 ; \mathrm{S}, 6.75$.

1,2-Dibromo-3,4,4-trichloro-1-(2-naphthylthio)-1,3-butadiene (8c). Yield 63\%; White solid, mp: 65-67 C. $\mathrm{R}_{f}$ (Petroleum ether): 0.71; IR ( $\left.\mathrm{KBr}, \mathrm{cm}^{-1}\right): 1585,1538(\mathrm{C}=\mathrm{C}), 3054\left(=\mathrm{C}-\mathrm{H}_{\text {arom }}\right)$; $\mathrm{UV}$-vis $\left(\mathrm{CHCl}_{3}\right): \lambda_{\max }(\log \varepsilon)=241(4.05) ;{ }^{1} \mathrm{H} \mathrm{NMR}\left(499.83 \mathrm{MHz}, \mathrm{CDCl}_{3}\right): \delta 7.16-7.92(\mathrm{~m}, 7 \mathrm{H}$, $\left.\mathrm{H}_{\text {arom}}\right) ;{ }^{13} \mathrm{C} \mathrm{NMR}\left(125.68 \mathrm{MHz}, \mathrm{CDCl}_{3}\right): \delta 110.75,118.94,122.85,125.97,126.28,126.44$, 126.84, 126.97, 127.80, 128.03, 129.43, 131.15, 132.94; $\mathrm{C}_{14} \mathrm{H}_{7} \mathrm{Br}_{2} \mathrm{Cl}_{3} \mathrm{~S}(\mathrm{M}, 473.44)$ Calcd. C, 35.52; H, 1.49; S, 6.77. Found C, 34.92; H, 1.03; S, 7.22.

1,2-Dibromo-3,4,4-trichloro-1-(1-octylthio)-1,3-butadiene (8e). Yield 65\%; Oil, $\mathrm{R}_{f}$ (Petroleum ether): 0.85; IR (film, $\left.\mathrm{cm}^{-1}\right): 2855,2927(\mathrm{C}-\mathrm{H}), 1538,1599(\mathrm{C}=\mathrm{C}), \mathrm{UV}$-vis $\left(\mathrm{CHCl}_{3}\right): \lambda_{\max }(\log \varepsilon)=$ $246 \mathrm{~nm}(5.3) ;{ }^{1} \mathrm{H}$ NMR (499.83 MHz, $\left.\mathrm{CDCl}_{3}\right): \delta 0.8\left(\mathrm{t}, J=7.32 \mathrm{~Hz}, 3 \mathrm{H}, \mathrm{CH}_{3}\right), 1.20-1.35(\mathrm{~m}$,

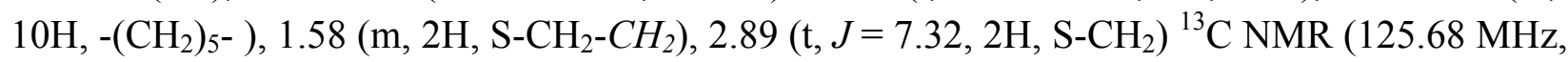


$\left.\mathrm{CDCl}_{3}\right): \delta 13.05\left(\mathrm{CH}_{3}\right) ; 21.61,27.46,28.00,28.04,28.08,30.74,35.76\left(\mathrm{CH}_{2}\right) ; 109.84,116.85$, 123.69, 128.43 ( $\left.\mathrm{C}_{\text {butadiene }}\right) ; \mathrm{C}_{12} \mathrm{H}_{17} \mathrm{Cl}_{3} \mathrm{Br}_{2} \mathrm{~S}$ (M, 459.50) Calcd. C, 31.37; H, 3.73; S, 6.98. Found C, 31.61; H, 3.49; S, 7.09.

1,2-Dibromo-3,4,4-trichloro-1-(1-decylthio)-1,3-butadiene (8f). Yield 78\%; Oil, $\mathrm{R}_{f}$ (Petroleum ether): 0.75; IR (film, cm $\left.{ }^{-1}\right): 2854,2924(\mathrm{C}-\mathrm{H}), 1538,1599(\mathrm{C}=\mathrm{C}), \mathrm{UV}-\mathrm{vis}\left(\mathrm{CHCl}_{3}\right): \lambda_{\max }(\log \varepsilon)=$ 244nm (5.1); ${ }^{1} \mathrm{H}$ NMR (499.83 MHz, $\left.\mathrm{CDCl}_{3}\right): \delta 0.81\left(\mathrm{t}, J=7.32 \mathrm{~Hz}, 3 \mathrm{H}, \mathrm{CH}_{3}\right), 1.19-1.34(\mathrm{~m}$, $\left.10 \mathrm{H},-\left(\mathrm{CH}_{2}\right)_{5^{-}}\right), 1.58\left(\mathrm{~m}, 2 \mathrm{H}, \mathrm{S}-\mathrm{CH}_{2}-\mathrm{CH}_{2}\right), 2.89\left(\mathrm{t}, J=7.32,2 \mathrm{H}, \mathrm{S}-\mathrm{CH}_{2}\right){ }^{13} \mathrm{C} \mathrm{NMR}(125.68 \mathrm{MHz}$, $\left.\mathrm{CDCl}_{3}\right): \delta 13.08\left(\mathrm{CH}_{3}\right) ; 21.65,28.05,28.08,28.27,28.42,28.49,28.62,30.87,35.75\left(\mathrm{CH}_{2}\right)$; 109.82, 116.85, 123.69, 128.48 ( $\left.\mathrm{C}_{\text {butadiene }}\right) ; \mathrm{C}_{14} \mathrm{H}_{21} \mathrm{Cl}_{3} \mathrm{Br}_{2} \mathrm{~S}(\mathrm{M}, 487.50)$ Calcd. C, 34.46; H, 4.30; S, 6.56. Found C, 34.09; H, 4.97; S, 7.08.

1,2-Dibromo-3,4,4-trichloro-1-(1-dodecylthio)-1,3-butadiene $\quad(\mathbf{8 g})$. Yield 92\%; Oil, $\mathrm{R}_{f}$ (Petroleum ether); 0.90; IR (film, $\mathrm{cm}^{-1}$ ): 1598, 1539 (C=C), 2854, $2954(\mathrm{C}-\mathrm{H})$. UV-vis $\left(\mathrm{CHCl}_{3}\right)$ : $\lambda_{\max }(\log \varepsilon)=243 \mathrm{~nm}(5.0) ;{ }^{1} \mathrm{H} \mathrm{NMR}\left(499.83 \mathrm{MHz}, \mathrm{CDCl}_{3}\right): \delta 0.82\left(\mathrm{t}, J=7.32 \mathrm{~Hz}, 3 \mathrm{H}, \mathrm{CH}_{3}\right)$, 1.19-1.34 (m, 26H, $\left.-\left(\mathrm{CH}_{2}\right)_{13^{-}}\right), 1.58\left(\mathrm{~m}, 2 \mathrm{H}, \mathrm{S}-\mathrm{CH}_{2}-\mathrm{CH}_{2}\right), 2.92\left(\mathrm{t}, J=7.32,2 \mathrm{H}, \mathrm{S}-\mathrm{CH}_{2}\right){ }^{13} \mathrm{C}$ NMR (125.68 MHz, $\left.\mathrm{CDCl}_{3}\right): \delta 13.09\left(\mathrm{CH}_{3}\right) ; 21.67,27.45,28.04,28.07,28.32,28.41,28.45$, 28.53, 28.61, 30.90, $35.75\left(\mathrm{CH}_{2}\right) ; 109.81,116.85,123.69,128.48$ ( $\left.\mathrm{C}_{\text {butadiene }}\right) ; \mathrm{C}_{16} \mathrm{H}_{25} \mathrm{Cl}_{3} \mathrm{Br}_{2} \mathrm{~S}(\mathrm{M}$, 515.50) Calcd. C, 37.24; H, 4.84; S, 6.20. Found C, 38.01; H, 4.85; S, 6.55.

1,2-Dibromo-3,4,4-trichloro-1-(1-hexadecylthio)-1,3-butadiene (8h). Yield 86\%; Oil, $\mathrm{R}_{f}$ (Petroleum ether); 0.86; IR (film, $\mathrm{cm}^{-1}$ ): $1598(\mathrm{C}=\mathrm{C}), 2853,2923(\mathrm{C}-\mathrm{H})$. UV-vis $\left(\mathrm{CHCl}_{3}\right): \lambda_{\max }$ $(\log \varepsilon)=245 \mathrm{~nm}(4.8) ;{ }^{1} \mathrm{H}$ NMR $\left(499.83 \mathrm{MHz} \mathrm{CDCl}_{3}\right): \delta 0.81\left(\mathrm{t}, J=7.32 \mathrm{~Hz}, 3 \mathrm{H}, \mathrm{CH}_{3}\right), 1.18-$ $1.35\left(\mathrm{~m}, 26 \mathrm{H},-\left(\mathrm{CH}_{2}\right)_{13^{-}}\right), 1.56\left(\mathrm{~m}, 2 \mathrm{H}, \mathrm{S}-\mathrm{CH}_{2}-\mathrm{CH}_{2}\right), 2.89\left(\mathrm{t}, J=7.32,2 \mathrm{H}, \mathrm{S}-\mathrm{CH}_{2}\right){ }^{13} \mathrm{C}$ NMR $\left(125.68 \mathrm{MHz}, \mathrm{CDCl}_{3}\right): \delta 13.09\left(\mathrm{CH}_{3}\right) ; 21.68,27.45,28.04,28.07,28.35,28.41,28.46,28.53$, 28.62, 28.65, 28.67, 28.68, 28.69, 30.92, $35.75\left(\mathrm{CH}_{2}\right)$; 109.82, 116.85, 123.68, 128.47 ( $\left.\mathrm{C}_{\text {butadiene }}\right)$; $\mathrm{C}_{20} \mathrm{H}_{33} \mathrm{Cl}_{3} \mathrm{Br}_{2} \mathrm{~S}$ (M, 571.50) Calcd. C, 42.03; H, 5.78; S, 5.60. Found C, 41.95; H, 5.85; S, 5.55.

1,2-Dibromo-3,4,4-trichloro-1-(1-otcadecylthio)-1,3-butadiene (8i). Yield 76\%; Oil, $\mathrm{R}_{f}$ (Petroleum ether); 0.80; IR (film, $\left.\mathrm{cm}^{-1}\right): 2855,2925(\mathrm{C}-\mathrm{H}), 1550(\mathrm{C}=\mathrm{C}) . \mathrm{UV}$-vis $\left(\mathrm{CHCl}_{3}\right): \lambda_{\max }$ $(\log \varepsilon)=244 \mathrm{~nm}(4.4) ;{ }^{1} \mathrm{H} \mathrm{NMR}\left(499.83 \mathrm{MHz}, \mathrm{CDCl}_{3}\right): \delta 0.81\left(\mathrm{t}, J=7.32 \mathrm{~Hz}, 3 \mathrm{H}, \mathrm{CH}_{3}\right), 1.18-$ $1.38\left(\mathrm{~m}, 30 \mathrm{H},-\left(\mathrm{CH}_{2}\right)_{15}\right), 1.58\left(\mathrm{~m}, 2 \mathrm{H}, \mathrm{S}-\mathrm{CH}_{2}-\mathrm{CH}_{2}\right), 2.92\left(\mathrm{t}, J=7.32,2 \mathrm{H}, \mathrm{S}-\mathrm{CH}_{2}\right){ }^{13} \mathrm{C}$ NMR $\left(125.68 \mathrm{MHz}, \mathrm{CDCl}_{3}\right): \delta 13.09\left(\mathrm{CH}_{3}\right) ; 21.68,27.46,27.53,28.04,28.08,28.35,28.42,28.46$, 28.53, 28.62, 28.63, 28.65, 28.67, 28.69, 28.76, 30.92, $35.76\left(\mathrm{CH}_{2}\right) ; 109.84,116.85,123.69$, 128.48 ( $\left.\mathrm{C}_{\text {butadiene }}\right) ; \mathrm{C}_{22} \mathrm{H}_{37} \mathrm{Cl}_{3} \mathrm{Br}_{2} \mathrm{~S}(\mathrm{M}, 599.77)$ Calcd. C, 44.05; H, 6.22; S, 5.34. Found C, 44.52; H, 6.35; S, 5.12.

1,2-Dibromo-3,4,4-trichloro-1-(1-hexadecylthio)-1,3-butadiene (11h). Yield 88\%; Oil, $\mathrm{R}_{f}$ (Petroleum ether); 0.83; IR (film, $\mathrm{cm}^{-1}$ ): $1594(\mathrm{C}=\mathrm{C}), 2853,2923(\mathrm{C}-\mathrm{H})$. UV-vis $\left(\mathrm{CHCl}_{3}\right): \lambda_{\max }$ $(\log \varepsilon)=244 \mathrm{~nm}(5.3) ;{ }^{1} \mathrm{H}$ NMR $\left(499.83 \mathrm{MHz} \mathrm{CDCl}_{3}\right): \delta 0.81\left(\mathrm{t}, J=7.32 \mathrm{~Hz}, 3 \mathrm{H}, \mathrm{CH}_{3}\right), 1.19-$ $1.35\left(\mathrm{~m}, 26 \mathrm{H},-\left(\mathrm{CH}_{2}\right)_{13^{-}}\right), 1.58\left(\mathrm{~m}, 2 \mathrm{H}, \mathrm{S}-\mathrm{CH}_{2}-\mathrm{CH}_{2}\right), 2.89\left(\mathrm{t}, J=7.32,2 \mathrm{H}, \mathrm{S}-\mathrm{CH}_{2}\right){ }^{13} \mathrm{C}$ NMR $\left(125.68 \mathrm{MHz}, \mathrm{CDCl}_{3}\right): \delta 13.11\left(\mathrm{CH}_{3}\right) ; 21.69,27.46,28.06,28.10,28.36,28.43,28.44,28.47$, 28.55, 28.63, 28.66, 28.68, 28.70, 30.93, $35.75\left(\mathrm{CH}_{2}\right)$; 109.58, 118.25, 123.64, 128.41 ( $\left.\mathrm{C}_{\text {butadiene }}\right)$; $\mathrm{C}_{20} \mathrm{H}_{33} \mathrm{Cl}_{2} \mathrm{Br}_{3} \mathrm{~S}$ (M, 616.36); Calcd. C, 38.93; H, 5.35; S, 5.19. Found C, 38.25; H, 5.69; S, 4.97. 


\section{General procedure 5}

Arylthiosubstituted butatriene compound $(1.5 \mathrm{mmol})$ and iodine $(0.38 \mathrm{~g}, 1.5 \mathrm{mmol})$ were stirred in $\mathrm{CCl}_{4}(30 \mathrm{~mL})$ for $3 \mathrm{~h}$ at room temperature. Ether was added to the reaction mixture and extracted with $100 \mathrm{ml}$ of $3 \%$ aqueous solution of $\mathrm{Na}_{2} \mathrm{~S}_{2} \mathrm{O}_{5}$ twice. The organic layer was separated, washed with water $(4 \times 30 \mathrm{~mL})$, dried with anhydrous $\mathrm{MgSO}_{4}$. The solvent was evaporated and the residue was purified by column chromatography over silica gel (petroleum ether/chloroform). 1-Chloro-2,3-diiodo-1,4,4-tris(4-bromophenylthio)-1,3-butadiene (15a). Yield 87\%; Oil, $\mathrm{R}_{f}$ (Petroleum ether): 0.35; IR (film, $\left.\mathrm{cm}^{-1}\right): 1563(\mathrm{C}=\mathrm{C}), 3077\left(=\mathrm{C}-\mathrm{H}_{\text {arom}}\right) .{ }^{1} \mathrm{H}$ NMR $(499.83 \mathrm{MHz}$, $\left.\mathrm{CDCl}_{3}\right): \delta 6.84-7.46\left(\mathrm{~m}, 12 \mathrm{H}, \mathrm{H}_{\text {arom}}\right) ;{ }^{13} \mathrm{C} \mathrm{NMR}\left(125.68 \mathrm{MHz}, \mathrm{CDCl}_{3}\right): \delta 61.92,67.46,99.76$, $102.30,102.54,102.79,121.41,121.65,121.70,122.58,129.37,129.86,130.19,130.47,130.49$, $130.55,130.60,130.69,130.73,130.76,130.80,131.50,131.54,131.61,132.20,132.30,133.07$, $133.13,133.18,133.39,133.84,133.92,134.18,138.46,139.45 ;\left(\mathrm{CH}_{\text {arom }}, \mathrm{C}_{\text {arom }}, \mathrm{C}_{\text {butadien }} \mathrm{e}\right) \mathrm{MS}$ (+ESI): $m / z 775.32[\mathrm{M}-\mathrm{I}]^{+} ; \mathrm{C}_{22} \mathrm{H}_{12} \mathrm{Br}_{3} \mathrm{ClI}_{2} \mathrm{~S}_{3}$ (M, 901.50); Calcd. C, 29.31; H, 1.34; S, 10.67. Found C, 29.88; H, 1.93; S, 11.09 .

2,3-Diiodo-1,1,1,4-tetrakis(4-bromophenylthio)-1,3-butadiene (16a). Yield 76\%; Light yellow crystalline solid, mp: $135-137{ }^{\circ} \mathrm{C} ; \mathrm{R}_{f}$ [Petroleum ether/ $\left.\mathrm{CCl}_{4}(1: 1)\right]: 0.42 ; \mathrm{IR}\left(\mathrm{KBr}, \mathrm{cm}^{-1}\right)$ : $1564(\mathrm{C}=\mathrm{C}), 3076\left(=\mathrm{C}-\mathrm{H}_{\text {arom }}\right) .{ }^{1} \mathrm{H}$ NMR $\left(499.83 \mathrm{MHz}, \mathrm{CDCl}_{3}\right): \delta 6.88-7.25\left(\mathrm{~m}, 16 \mathrm{H}, \mathrm{H}_{\text {arom }}\right) ;{ }^{13} \mathrm{C}$ NMR (125.68 MHz, $\left.\mathrm{CDCl}_{3}\right): \delta 131.98,132.02,133.58,134.16\left(\mathrm{CH}_{\text {arom }}\right), 68.40,106.04,122.67$, 122.74, 131.81, 139.28 ( $\mathrm{C}_{\text {arom }}$ and $\left.\mathrm{C}_{\text {butadiene }}\right)$; $\mathrm{MS}(+\mathrm{ESI}): \mathrm{m} / \mathrm{z} 1052[\mathrm{M}]^{+} ; \mathrm{C}_{28} \mathrm{H}_{16} \mathrm{Br}_{4} \mathrm{I}_{2} \mathrm{~S}_{4}(\mathrm{M}$, 1054.11). Calcd. C, 31.90; H, 1.53; S, 12.17. Found C, 32.23; H, 1.75; S, 12.88.

\section{Acknowledgements}

We thank the Research Fund of Mustafa Kemal University and the Research Fund of Istanbul Univesity for financial support for this work.

\section{References}

1. Roedig, A.; Zaby G.; Scharf, W. Chem. Ber. 1977, 110, 1484.

2. Roedig, A.; Zaby, G. Chem. Ber. 1979, 112, 1614.

3. Roedig, A.; İbiş, C.; Zaby, G. Chem. Ber. 1981, 114, 684.

4. İbiş, C. Chim. Acta Turc. 1983, 11(2), 253.

5. İbiş, C. Liebigs Ann. Chem. 1984, 1873.

6. İbiş, C. Liebigs Ann. Chem. 1987, 1009.

7. İbiş, C.; Sahinler Ayla, S. Arkivoc 2008, (xvi), 29.

8. Diamond Alkali Company (Ert. H. Bluestone), US Patent 302127013 Feb. 1962.

9. Hankp, R.; Hammond, M. D.; Fruchtmann, R.; Pfitzner, J.; Place, G. A. J. Med. Chem. 1990, 33, 1163. 
10. Carothers, W. H.; Williams, I.; Collins, A. M.; Kirby, J. E. J. Am. Chem. Soc. 1931, 53, 4203.

11. Tebbe, M. J.; Jensen, C. B.; Spitzer, W. A.; Franklin, R. B.; George, M. H.; Phillips, D. L. Antiviral Res. 1999, 42(1), 25.

12. Pollack,S.K.; Fiseha, A.; Narayanswamy, B. Macromolecules 1997, 30, 5265.

13. Gingras, M.; Raimundo, J. M.; Chabre, Y. M. Angew. Chem. Int. Ed. 2006, 45, 1686.

14. Schmidt, A.; Rahimi, A.; Gjikaj, M. Synthesis 2009, 14, 2371.

15. Block, E.; Tries, F.; He, C.; Guo, C.; Thiruvazhi, M.; Toscano, P. J. Org. Lett. 2003, 5, 1325. 